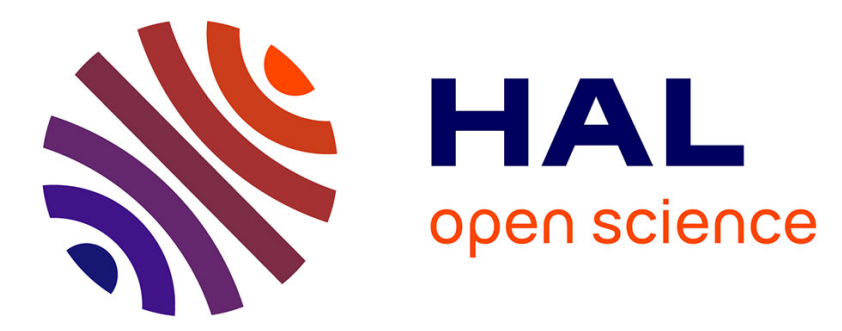

\title{
Magnetocardiography with sensors based on giant magnetoresistance
}

M. Pannetier-Lecoeur, L. Parkkonen, N. Sergeeva-Chollet, H. Polovy, C. Fermon, C. Fowley

\section{- To cite this version:}

M. Pannetier-Lecoeur, L. Parkkonen, N. Sergeeva-Chollet, H. Polovy, C. Fermon, et al.. Magnetocardiography with sensors based on giant magnetoresistance. Applied Physics Letters, 2011, 98, pp.153705. 10.1063/1.3575591 . cea-01890264

\section{HAL Id: cea-01890264 https://hal-cea.archives-ouvertes.fr/cea-01890264}

Submitted on 8 Oct 2018

HAL is a multi-disciplinary open access archive for the deposit and dissemination of scientific research documents, whether they are published or not. The documents may come from teaching and research institutions in France or abroad, or from public or private research centers.
L'archive ouverte pluridisciplinaire HAL, est destinée au dépôt et à la diffusion de documents scientifiques de niveau recherche, publiés ou non, émanant des établissements d'enseignement et de recherche français ou étrangers, des laboratoires publics ou privés. 


\title{
Magnetocardiography with sensors based on giant magnetoresistance
}

\author{
M. Pannetier-Lecoeur, L. Parkkonen, N. Sergeeva-Chollet, H. Polovy, C. Fermon, and C. Fowley
}

Citation: Appl. Phys. Lett. 98, 153705 (2011); doi: 10.1063/1.3575591

View online: https://doi.org/10.1063/1.3575591

View Table of Contents: http://aip.scitation.org/toc/apl/98/15

Published by the American Institute of Physics

\section{Articles you may be interested in}

Magnetocardiogram measured by fundamental mode orthogonal fluxgate array

Journal of Applied Physics 117, 17B322 (2015); 10.1063/1.4918958

Tunneling magnetoresistance sensor with $\mathrm{pT}$ level $1 / \mathrm{f}$ magnetic noise

AIP Advances 7, 056676 (2017); 10.1063/1.4978465

Low-frequency noise measurements on commercial magnetoresistive magnetic field sensors

Journal of Applied Physics 97, 10Q107 (2005); 10.1063/1.1861375

Tunnel magnetoresistance of $604 \%$ at $300 \mathrm{~K}$ by suppression of Ta diffusion in $\mathrm{CoFe} B / \mathrm{MgO} / \mathrm{CoFe} B$ pseudospin-valves annealed at high temperature

Applied Physics Letters 93, 082508 (2008); 10.1063/1.2976435

Progress toward a thousandfold reduction in $1 / f$ noise in magnetic sensors using an ac microelectromechanical system flux concentrator (invited)

Journal of Applied Physics 99, 08B317 (2006); 10.1063/1.2170067

Magnetoencephalography with an atomic magnetometer

Applied Physics Letters 89, 211104 (2006); 10.1063/1.2392722

\section{Conference Proceedings}

Get $30 \%$ off all print proceedings! 


\title{
Magnetocardiography with sensors based on giant magnetoresistance
}

\author{
M. Pannetier-Lecoeur, ${ }^{1, a)}$ L. Parkkonen, ${ }^{1, b)}$ N. Sergeeva-Chollet, ${ }^{1}$ H. Polovy, ${ }^{1}$ C. Fermon, ${ }^{1}$ \\ and C. Fowley ${ }^{2}$ \\ ${ }^{1}$ DSM/IRAMIS/SPEC, CNRS URA 2464, CEA Saclay, 91191 Gif-sur-Yvette, France \\ ${ }^{2}$ CRANN, Trinity College, Dublin 2, Ireland
}

(Received 19 January 2011; accepted 10 March 2011; published online 14 April 2011)

\begin{abstract}
Biomagnetic signals, mostly due to the electrical activity in the body, are very weak and they can only be detected by the most sensitive magnetometers, such as Superconducting Quantum Interference Devices (SQUIDs). We report here biomagnetic recordings with hybrid sensors based on Giant MagnetoResistance (GMR). We recorded magnetic signatures of the electric activity of the human heart (magnetocardiography) in healthy volunteers. The P-wave and QRS complex, known from the corresponding electric recordings, are clearly visible in the recordings after an averaging time of about $1 \mathrm{~min}$. Multiple recordings at different locations over the chest yielded a dipolar magnetic field map and allowed localizing the underlying current sources. The sensitivity of the GMR-based sensors is now approaching that of SQUIDs and paves way for spin electronics devices for functional imaging of the body. (C) 2011 American Institute of Physics. [doi:10.1063/1.3575591]
\end{abstract}

Many living organisms generate weak but measurable magnetic fields. These biomagnetic signals can be either static fields due to dc currents or small magnetic particles in the tissues or low-frequency fields due to transient or oscillatory electric activity. In humans, the two most studied biomagnetic signal sources are the brain (magnetoencephalography or MEG) and the heart (magnetocardiography or MCG). MEG and MCG signals result from the electric currents flowing in active nerve cells in the brain and cardiac muscle, respectively. The temporal accuracy of these measurements is very high (in the ms range), and recording the signals at multiple locations typically allows localizing the generators.

MEG and MCG are completely noninvasive and even contactless, allowing measurements without the use of electrodes, which is a benefit when studying infants, for example. Furthermore, unlike electric signals, magnetic signals are not distorted by the conductivity changes between different tissues.

Since biomagnetic signals are very weak, typically few tens of picoteslas $\left(1 \mathrm{pT}=10^{-12} \mathrm{~T}\right)$ for MCG and few tens of femtoteslas ( $1 \mathrm{fT}=10^{-15} \mathrm{~T}$ ) for MEG, only few sensor types have been used so far for measuring them. Superconducting Quantum Interference Devices (SQUIDs) ${ }^{1}$ have been the sensor of choice for MEG (Ref. 2) and MCG, ${ }^{3}$ thanks to their exquisite sensitivity at low frequencies. Commercial MEG systems employ liquid Helium-cooled low- $T_{\mathrm{c}} \mathrm{Nb}$-based SQUIDs, which can reach sensitivities of few $\mathrm{fT} / \sqrt{ } \mathrm{Hz}$ down to $1 \mathrm{~Hz}$ and can be manufactured with reproducible properties. High- $T_{\mathrm{c}}$ SQUIDs have been used also for biomagnetic recordings ${ }^{4,5}$ but their application has been limited by the order-of-magnitude larger noise levels, and by the difficulties in realizing durable Josephson junctions in high- $T_{\mathrm{c}}$ superconducting material.

Several emerging sensor technologies for detecting extremely weak magnetic fields at low frequencies have been

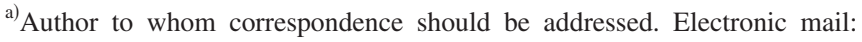
myriam.lecoeur@cea.fr.

${ }^{b)}$ Also at Elekta Oy, Helsinki, Finland.
}

proposed during the past few years. Spin exchange relaxation-free atomic magnetometers exhibit femtotesla sensitivity ${ }^{6}$ and recordings of biomagnetic signals have been demonstrated. ${ }^{7,8}$

Thin-film technology allows precise fabrication of arrays of sensors using patterning techniques developed for semiconductor industry. Exploiting this technology and spin electronics, we have previously developed a magnetometer whose sensitivity reaches the femtotesla range. ${ }^{9}$ This sensor combines a spin valve to a superconducting flux-to-field transformer and can achieve white noise levels of a few femtoteslas. As the sensor does not contain a junction, the superconducting loop can be made either of low- or high- $T_{\mathrm{c}}$ material without difficulty.

A magnetic field applied to the superconducting loop gives rise to a supercurrent which flows in the loop to counteract the applied field. Part of the loop is narrowed [see Fig. 1(a)] to increase the local current density, resulting in a local amplification of the applied field by the ratio of the loop size and constriction width. The amplified field can be detected by magnetoresistive sensors located on top or below the constriction. Giant MagnetoResistance (GMR) (Refs. 10 and 11) and Tunnel MagnetoResistance (TMR) (Refs. 12 and 13) devices are suitable because of their high sensitivity to in-plane fields.

Our sensor comprised four GMR spin valves with the following composition: $\mathrm{Ta}(5) / \mathrm{NiFe}(3.5) / \mathrm{CoFe}(1.5) /$ $\mathrm{Cu}(2.9) / \mathrm{CoFe}(2.5) / \operatorname{IrMn}(10) / \mathrm{Ta}(5)$; the thicknesses are in nanometers. The four GMR elements were patterned by conventional photolithography in a yoke shape to prevent the formation of magnetic domains in the sensing area. ${ }^{14}$ The GMRs were configured as a Wheatstone bridge. The large Niobium loop was deposited by sputtering. The GMR stacks and the loop were electrically isolated by a $300-\mathrm{nm} \mathrm{Si}_{3} \mathrm{~N}_{4}$ layer. For the MCG measurements, the sensor was located at the bottom of a fiberglass dewar and immersed in liquid helium.

The GMRs were biased with a current of about $0.3 \mathrm{~mA}$. The sensitivity of the mixed sensor was of $6000 \mathrm{~V} / \mathrm{T}$ for this bias current; the resulting voltage was measured by a low- 
(a)

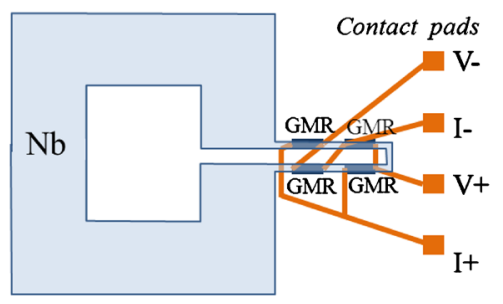

(b)

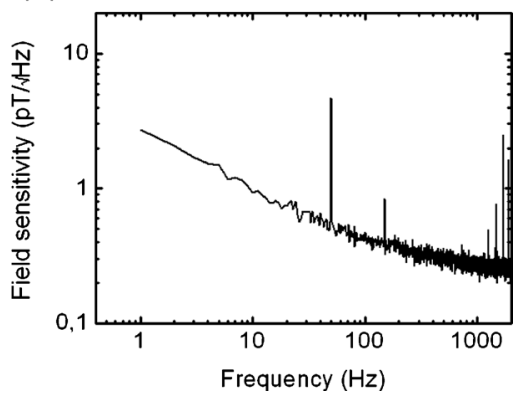

FIG. 1. (Color online) (a) Schematic diagram of the sensor: four GMR elements are mounted on a constriction of the large Niobium loop. The GMRs are wired to form a Wheatstone bridge. Dimensions are not to scale. (b) Field sensitivity at $4 \mathrm{~K}$ extracted from the noise power spectrum of the mixed sensor. Note that in the thermal noise $(\mathrm{f}>1 \mathrm{kHz})$, the limitation is due to the preamplifier noise, which is of $1.7 \mathrm{nV} / \sqrt{\mathrm{Hz}}$ (and corresponds to $200 \mathrm{fT} / \sqrt{\mathrm{Hz}}$ ). Power line peak at $50 \mathrm{~Hz}$ is well visible on the graph. noise preamplifier stage with a gain of 500 (custom-built around INA103 with a voltage noise level of $1.2 \mathrm{nV} / \sqrt{ } \mathrm{Hz}$ ) operating at room temperature. In the thermal noise, the sensitivity is limited by the preamplifier noise, leading to an equivalent field sensitivity of $200 \mathrm{fT} / \sqrt{ } \mathrm{Hz}$. However, at lowfrequency the sensitivity is limited by the $1 / \mathrm{f}$ noise of the GMRs and gives a noise of $17 \mathrm{nV} / \sqrt{ } \mathrm{Hz}$ at $1 \mathrm{~Hz}$, which corresponds to $3 \mathrm{pT} / \sqrt{ } \mathrm{Hz}$ at $1 \mathrm{~Hz}$ [see Fig. 1(b)]. Secondary amplification (gain 100) and filtering (pass-band 0.1-300 $\mathrm{Hz}$ ) was performed by a Stanford Research Systems (Sunnyvale, CA, USA) model 560 low-noise amplifier. The signal was then fed to an extra analog input of the acquisition system of a commercial 306-channel MEG system (Elekta Oy, ${ }^{15}$ Helsinki, Finland). An electrocardiographic (ECG) signal was acquired simultaneously by a pair of surface electrodes attached to the wrists of the subject and connected to a bipolar channel of the EEG amplifier and acquisition system of the Elekta MEG device. The MCG and ECG signals were filtered to $0.1-330 \mathrm{~Hz}$ and sampled simultaneously at $1 \mathrm{kHz}$. The acquisition system also provided the possibility for online monitoring of the signals (Fig. 2).

Since the MCG signals are on the order of a few tens of pT, external magnetic interference can easily mask them, particularly when using a magnetometer sensor, whichunlike a gradiometer-does not provide any additional attenuation of remote sources. Therefore, we performed the recordings in the magnetically shielded room (MSR) of the MEG facility of the NeuroSpin center of CEA/Saclay, France. The MSR (MaxShield ${ }^{\mathrm{TM}}$ by Elekta $\mathrm{Oy}^{15}$ ) comprised a single shell made of interleaved mu-metal and Aluminum plates. The internal active compensation system was not used.

The subject laid down on his/her back, with chest right below the dewar but not touching it. The distance between the sensor and the skin was $25-30 \mathrm{~mm}$. The dewar was

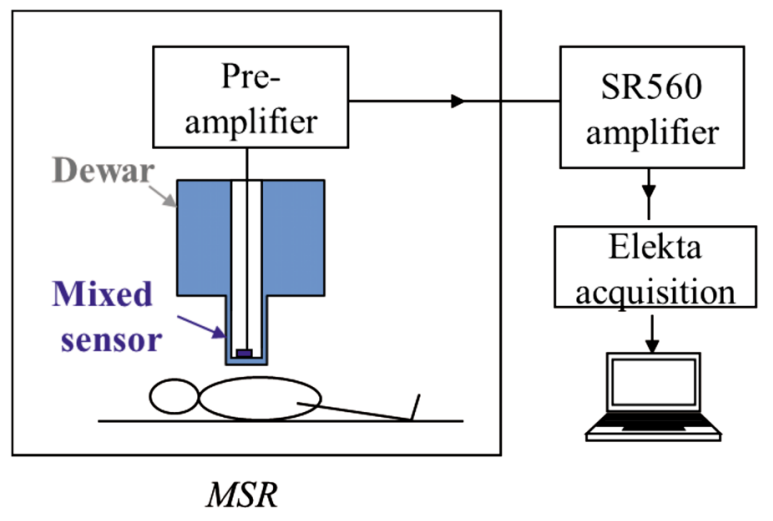

FIG. 2. (Color online) The experimental setup (ECG not shown here). manually translated in the horizontal plane to cover a 6-by-6 point grid of measurement locations. The spacing was $3 \mathrm{~cm}$ in the left-right direction and $5 \mathrm{~cm}$ in the foot-head direction (except that the lowest rows were separated only by 2 and 3 $\mathrm{cm}$ due to space constraints), and the grid was displaced to the left of the subject by $1.5 \mathrm{~cm}$; the convention was adapted from the work of Saarinen et al. ${ }^{16}$ During the measurement, the dewar position was read with respect to a reference point using two scales, and these positions were later inserted in the data file to enable estimating field maps and source locations.

The MCG signal was recorded for about one minute per location, together with the ECG. The resulting 36 continuous recordings for each subject were then separately averaged by using the QRS peak onset of the conditioned ECG signal as the trigger and by rejecting epochs with excessively large $(>60 \mathrm{pT})$ signal variation due to external perturbation. The number of clean epochs varied from 46 to 54 per location: the variation was due to the rejections. The averages were merged into one multi-channel file that contained the dewar positions as the channel locations. The characteristic components of normal ECG, the P-wave, QRS complex and T-wave, ${ }^{16,17}$ are all present in our magnetic data; see Fig. 3 . The spatial variation in the signal shape is also clearly visible, demonstrating the added value of magnetic recordings with respect to ECG.

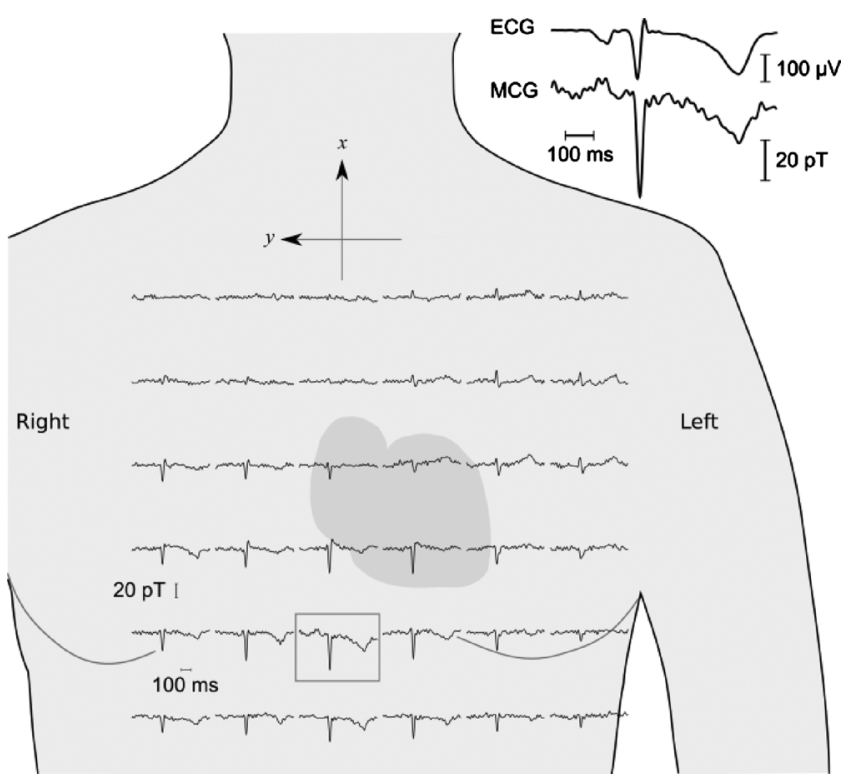

FIG. 3. MCG signals measured at multiple locations on a single subject. The enclosed MCG trace is shown enlarged with the ECG signal. Pass-band $0.1-40 \mathrm{~Hz}$. The position of the traces with respect to the heart is only approximate. 


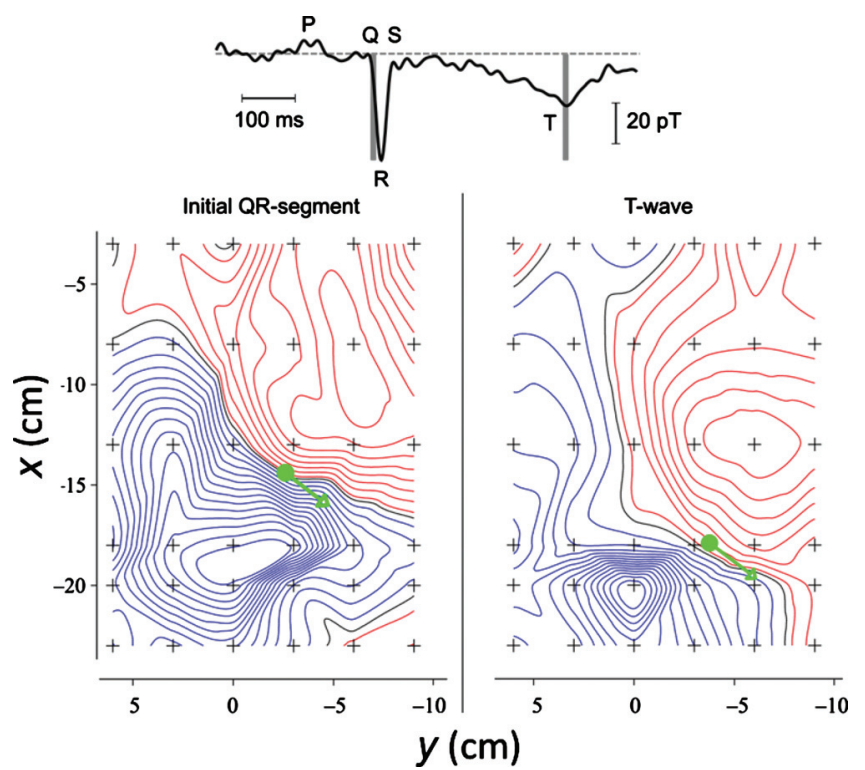

FIG. 4. (Color) Isocontours of the normal component of the magnetic field during the initial QR-segment and T-wave; blue and red indicate field in and out, respectively, of the chest of the subject. The green arrows denote the fitted equivalent current dipoles.

The magnetic field map (Fig. 4) shows the polarity inversion for the initial QR-segment approximately along the diagonal of the measurement grid, suggesting a dipolar source with a similar orientation. This pattern changes for the $\mathrm{T}$-wave, indicating a partially separate generator.

We performed simple equivalent current dipole modeling of the MCG components using conducting half space as the conductor model. For the initial QR-segment, R-peak, and T-wave, the fitted dipoles explained $84 \%, 97 \%$, and $84 \%$ (goodness of fit) of the spatial variation in the signal pattern and yielded dipole moments of $0.82 \mu \mathrm{Am}, 3.0 \mu \mathrm{Am}$, and $1.6 \mu \mathrm{Am}$, respectively. Figure 4 shows the QR-segment and $\mathrm{T}$-wave dipole locations and orientations projected on the sensor plane.

In summary, we have performed biomagnetic measurements with GMR-based sensors. We recorded MCG signals at multiple locations on a healthy volunteer, estimated a magnetic field map, and succeeded in modeling the signal patterns with equivalent current dipoles with a small residual variance. Further improvements in the setup, including the use of TMR elements for the field sensing, and reducing the distance from the sensor to the room temperature surface of the dewar, may allow detection of MEG responses which are orders of magnitude weaker than MCG signals.

With mixed sensors, biomagnetic measurements might become feasible at liquid nitrogen temperature, which would strongly reduce the cost and complexity of MCG and MEG systems. Furthermore, as these sensors can also be used for MRI in low field $(<15 \mathrm{mT}){ }^{18}$ they could offer the possibility of obtaining anatomical and functional information of the heart and the brain with the same apparatus.

We thank Dr. Jukka Nenonen for useful discussions and Dr. Denis Le Bihan for his help in setting up the medical protocol of the Neurospin experiment. This work has been partially supported by the European Community through the MEGMRI project (Grant No. HEALTH-F5-2008-200859) in the FP7 program. Informed consents were obtained from each participant in accordance with the Declaration of Helsinki (2008) and the Ethics Committee on Human Research at the Commissariat à l'Energie Atomique et aux Energies Alternatives (CEA/DSV/I2BM/NeuroSpin, Gif-sur-Yvette, France).

${ }^{1}$ J. Clarke and A. I. Braginski, The SQUID Handbook (Wiley, New York, 2004), Vol. I.

${ }^{2}$ O. V. Lounasmaa and H. Seppä, J. Low Temp. Phys. 135, 295 (2004).

${ }^{3}$ M. M. Budnyk, I. D. Voytovych, Y. D. Minov, P. G. Sutkovyi, M. A. Primin, I. V. Nedayvoda, and V. V. Vasyliev, Neuropsychiatry Neuropsychol Behav. Neurol. 2004, 112 (2006).

${ }^{4}$ K. A. Kouznetsov, J. Borgmann, and J. Clarke, Appl. Phys. Lett., 75, 1979-1981 (1999).

${ }^{5}$ Y. Zhang, G. Panaitov, S. G. Wang, N. Wolters, R. Otto, J. Schubert, W. Zander, H.-J. Krause, H. Soltner, H. Bousack, and A. I. Braginski, Appl. Phys. Lett. 76, 906 (2000).

${ }^{6}$ K. Kominis, T. W. Kornack, J. C. Allred, and M. V. Romalis, Nature (London) 422, 596 (2003).

${ }^{7}$ H. Xia, A. Ben-Amar Baranga, D. Hoffman, and M. V. Romalis, Appl. Phys. Lett. 89, 211104 (2006).

${ }^{8}$ S. Knappe, T. H. Sander, O. Kosch, F. Wiekhorst, J. Kitching, and L. Trahms, Appl. Phys. Lett. 97, 133703 (2010).

${ }^{9}$ M. Pannetier, C. Fermon, G. Le Goff, J. Simola, and E. Kerr, Science 304, 1648 (2004)

${ }^{10}$ M. Baibich, J. M. Broto, A. Fert, F. Nguyen, V. D. F. Petroff, P. Etienne, G. Creuzet, A. Friederich, and J. Chazelas, Phys. Rev. Lett. 61, 2472 (1988).

${ }^{11}$ P. P. Freitas, R. Ferreira, S. Cardoso, and F. Cardoso, J. Phys.: Condens. Matter 19, 165221 (2007).

${ }^{12}$ M. Julliere, Phys. Lett. A 54, 225 (1975).

${ }^{13}$ J. S. Moodera, L. R. Kinder, T. M. Wong, and R. Meservey, Phys. Rev. Lett. 74, 3273 (1995).

${ }^{14}$ C. Fermon, M. Pannetier-Lecoeur, N. Biziere, and B. Cousin, Sens. Actuators, A 129, 203 (2006).

${ }^{15}$ Elekta Oy, Helsinki, Finland. Web: www.elekta.com.

${ }^{16}$ M. Saarinen, P. Karp, T. Katila, and P. Siltanen, Ann. Clin. Res. 10, 1 (1978).

${ }^{17}$ A. Kandori, K. Ogata, T. Miyashita, Y. Watanabe, K. Tanaka, M. Murakami, Y. Oka, H. Takaki, S. Hashimoto, Y. Yamada, K. Komamura, W. Shimizu, S. Kamakura, S. Watanabe, and I. Yamaguchi, Ann. Noninvasive Electrocardiol. 13, 391 (2008)

${ }^{18}$ N. Sergeeva-Chollet, H. Dyvorne, J. Dabek, Q. Herreros, H. Polovy, G. Le Goff, G. Cannies, M. Pannetier-Lecoeur, and C. Fermon, "Low field MRI with magnetoresistive mixed sensors," J. Phys.: Conf. Ser. (to be published). 